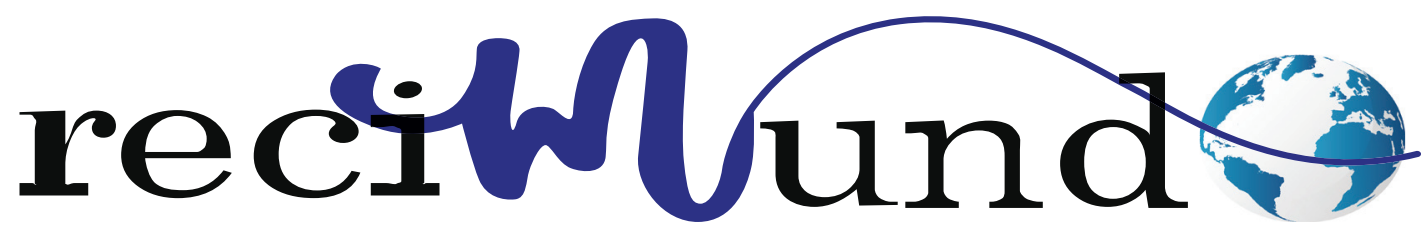

Revista Científica Mundo de la Investigación y el Conocimiento

DOI: 10.26820/recimundo/4.(4).octubre.2020.13-21

URL: http://recimundo.com/index.php/es/article/view/877

EDITORIAL: Saberes del Conocimiento

REVISTA: RECIMUNDO

ISSN: 2588-073X

TIPO DE INVESTIGACIÓN: Artículo de Revisión

CÓDIGo UNESCO: 32 Ciencias Médicas; 3201 Ciencias Clínicas

PAGINAS: $13-21$

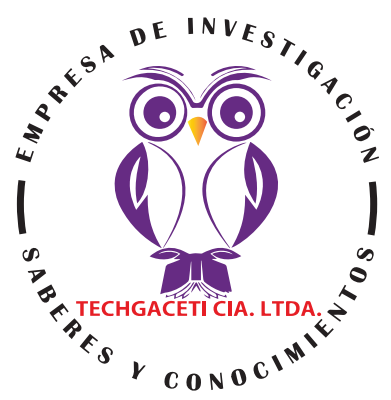

\title{
El paciente quemado y la insuficiencia renal aguda (IRA)
}

The burn patient and acute renal failure (ARF)

0 paciente queimado e o fracasso renal agudo (ARF)

Gema Paola Zambrano Andrade'; Madelaine Elizabeth Lascano Torres²; Ayrianna Katiusca Galán Velasco3; Dania Giomara Sinche Cruz ${ }^{4}$

RECIBIDO: 10/07/2020 ACEPTADO: 26/08/2020 PUBLICADO: 15/10/2020

1. Magister en Prevención de Riesgos Laborales; Médico General en funciones Hospitalarias; Hospital General Monte Sinaí; Guayaquil, Ecuador; gemipaozam@gmail.com; (iD https://orcid.org/0000-0002-4151-031X

2. Medico General; Distrito 09d12 Balao-Naranjal; Guayaquil, Ecuador; made.lascano@gmail.com; (iD https://orcid.org/00000002-8163-3744

3. Médica; Centro de Salud Taura; Distrito 09D12; Balao Naranjal, Guayaquil Ecuador; ayrianna_galanvelasco@hotmail.com; (iD) https://orcid.org/0000-0002-9171-8805

4. Médico General; Investigador Independiente; Guayaquil, Ecuador; dania_sinche95@hotmail.com; (iD https://orcid. org/0000-0002-7121-7755

CORRESPONDENCIA

Gema Paola Zambrano Andrade

marelejh@hotmail.com

Guayaquil, Ecuador

(C RECIMUNDO; Editorial Saberes del Conocimiento, 2020 


\section{RESUMEN}

Las quemaduras constituyen un problema de salud pública mundial, que tiene gran relevancia para la práctica médica, no sólo por su frecuencia sino por las consecuencias que produce sobre las zonas quemadas (locales) y aquellas complicaciones relacionadas con otros órganos (sistémicas). La Insuficiencia Renal Aguda (IRA) es una alteración o complicación renal que se presenta con frecuencia en el paciente quemado, la cual consiste es la disminución rápida de la función renal en días o semanas que causa la acumulación de productos nitrogenados en la sangre (azoemia) con o sin reducción de la diuresis. Las incidencias reportadas van desde 1\% a 40\%, y la mortalidad referida va desde $50 \%$ a $100 \%$. El objetivo del presente estudio consiste en compendiar de manera específica las diferentes alteraciones que pueden producirse en el sistema renal del paciente quemado, incluyendo la insuficiencia renal aguda (IRA), así como su manejo y monitoreo con el propósito de evitar mayores complicaciones. El modelo de investigación es una revisión de tipo documental bibliográfico. El fracaso renal que se observa durante los primeros días en el paciente quemado, suele ser de causa prerrenal por hipoperfusión. La insuficiencia renal aguda (IRA) es el resultado de una reducción del gasto cardíaco y del aumento en la secreción de mediadores inflamatorios (angiotensina, aldosterona y vasopresina). En conclusión, la IRA es una alteración del sistema renal que puede estar presente como patología pre existente en el paciente quemado o que puede producirse como consecuencia de las alteraciones sistémicas a las que es sometido el organismo tras la quemadura. Ambas condiciones son de vital importancia para el establecimiento de un manejo adecuado y reducir la tasa de morbimortalidad con respecto a estos pacientes.

Palabras clave: Paciente, quemado, insuficiencia, renal, aguda.

\section{ABSTRACT}

Burns are a global public health problem, which is highly relevant to medical practice, not only because of its frequency but also because of the consequences it produces on burned areas (local) and those complications related to other organs (systemic). Acute Renal Insufficiency (ARF) is a kidney disorder or complication that occurs frequently in burned patients, which consists of the rapid decrease in kidney function over days or weeks that causes the accumulation of nitrogenous products in the blood (azotemia ) with or without reduction in urine output. The reported incidences range from 1\% to $40 \%$, and the referred mortality ranges from $50 \%$ to $100 \%$. The objective of this study is to specifically summarize the different alterations that may occur in the renal system of the burned patient, including acute renal failure (ARF), as well as its management and monitoring in order to avoid further complications. The research model is a bibliographic documentary type review. The renal failure that is observed during the first days in the burned patient is usually of prerenal cause due to hypoperfusion. Acute renal failure (ARF) is the result of a reduction in cardiac output and an increase in the secretion of inflammatory mediators (angiotensin, aldosterone, and vasopressin). In conclusion, ARF is an alteration of the renal system that may be present as a pre-existing pathology in the burned patient or that may occur as a consequence of the systemic alterations to which the body is subjected after the burn. Both conditions are of vital importance for the establishment of an adequate management and to reduce the morbidity and mortality rate with respect to these patients.

Keywords: Patient, burned, insufficiency, renal, acute.

\section{RESUMO}

s queimaduras são um problema de saúde pública global, de grande relevância para a prática médica, não só pela frequência, mas também pelas consequências que produz nas áreas queimadas (locais) e nas complicações relacionadas com outros órgãos (sistémicas). A Insuficiência Renal Aguda (IRA) é um distúrbio ou complicação renal que ocorre com frequência em pacientes queimados, que consiste na rápida diminuição da função renal ao longo de dias ou semanas que causa o acúmulo de produtos nitrogenados no sangue (azotemia) com ou sem redução de saída de urina. As incidências relatadas variam de $1 \%$ a $40 \%$, e a mortalidade referida varia de 50\% a 100\%. O objetivo deste estudo é resumir especificamente as diferentes alterações que podem ocorrer no sistema renal do paciente queimado, incluindo insuficiência renal aguda (IRA), bem como seu manejo e monitoramento para evitar complicações posteriores. O modelo de pesquisa é uma revisão bibliográfica do tipo documental. A insuficiência renal observada nos primeiros dias no paciente queimado costuma ser de causa pré-renal por hipoperfusão. A insuficiência renal aguda (IRA) é o resultado de uma redução no débito cardíaco e um aumento na secreção de mediadores inflamatórios (angiotensina, aldosterona e vasopressina). Concluindo, a IRA é uma alteração do sistema renal que pode estar presente como uma patologia pré-existente no paciente queimado ou que pode ocorrer como consequência das alterações sistêmicas a que o corpo é submetido após a queimadura. Ambas as condições são de vital importância para o estabelecimento de um manejo adequado e para reduzir a morbimortalidade desses pacientes.

Palavras-chave: Paciente, queimado, insuficiência renal aguda. 


\section{Introducción}

A los fines de llevar a cabo el presente esLas quemaduras constituyen un problema de salud pública mundial, que tiene gran relevancia para la práctica médica, no sólo por su frecuencia sino por las consecuencias que produce sobre las zonas quemadas (locales) y aquellas complicaciones relacionadas con otros órganos (sistémicas).

Una quemadura es una lesión en la piel u otro tejido orgánico causada principalmente por el calor o debido a la radiación, radiactividad, electricidad, fricción o contacto con productos químicos. Se estima que 180.000 muertes cada año son causadas por quemaduras; la gran mayoría ocurre en países de ingresos bajos y medianos. En 2.004, casi 11 millones de personas en todo el mundo sufrieron quemaduras lo suficientemente graves como para requerir atención médica. (Organización Mundial de la Salud - OMS, 2018)

Clasificar una quemadura es sumamente importante para su tratamiento y pronóstico. Una de sus clasificaciones puede ser según su profundidad, en cuyo caso se clasifican en quemaduras de espesor parcial superficial y profunda y espesor total, asimismo pueden ser clasificadas según el porcentaje de la superficie corporal total afectada. Dentro de los principales factores que contribuyen a las complicaciones sistémicas tenemos la rotura de la piel y la integridad y pérdida de líquidos. A mayor "el porcentaje de la superficie corporal total (SCT) afectada, mayor es el riesgo de desarrollar complicaciones sistémicas. Los factores de riesgo de complicaciones sistémicas graves y mortalidad incluyen quemaduras de segundo y tercer grado $\geq 40 \%$ de la SCT". (Wolf, 2018)

Para Valdés, Palacios, \& Mariño (2015) la quemadura grave es el "tipo de agresión biológica más severa que puede sufrir el organismo y pone en peligro la vida del paciente por la permanencia del riesgo de sepsis y la falla multisistémica progresiva". (p. 131)

En el paciente con grandes quemaduras todos los sistemas del cuerpo, de una forma $u$ otra, se ven afectados. Ramírez, Ramírez B., González, Ramírez, \& Vélez (2010) afirman que dentro de las principales complicaciones del paciente quemado se encuentran: alteraciones inmunológicas, metabolicoendocrinas, gastrointestinales, hidroelectrolíticas, pulmonares, hematológicas, cardiovasculares y renales. (p. 55, 63)

La Insuficiencia Renal Aguda (IRA) es una alteración o complicación renal que se presenta en el paciente quemado. La insuficiencia renal aguda (IRA) "es la disminución rápida de la función renal en días o semanas que causa la acumulación de productos nitrogenados en la sangre (azoemia) con o sin reducción de la diuresis". (Malkina, 2019)

Sánchez \& de Jesús, 2017 citados por Sanchez, Barrazueta, Cisneros, \& Vaca (2019) acerca de la insuficiencia renal aguda (IRA) refieren:

Es una complicación frecuente en pacientes quemados, así como en los pacientes críticos con incidencias reportadas que van desde $1 \%$ a $40 \%$, y la mortalidad referida va desde $50 \%$ a $100 \%$. La mortalidad asociada a IRA sigue siendo muy alta en estos pacientes a pesar de los avances de cuidados intensivos y terapia de reemplazo renal (TRR). La incidencia de pacientes con IRA que necesitan TRR ha sido hasta un 50\% en pacientes quemados. (p. 173).

Asimismo, al inicio de la quemadura los reportes hablan de una incidencia de 1,3\% a $15,3 \%$ de insuficiencia renal, y esta variación es grande porque se analizaron múltiples series estadísticas de pacientes con quemaduras de más de 15\%. Los protocolos son de varios países con diferentes

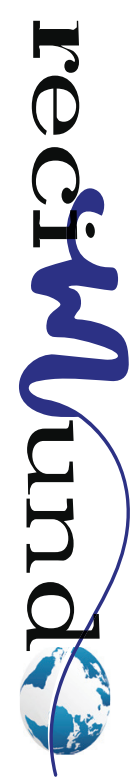


niveles de evidencia. (Ferrada, Tróchez, \& Ayala, 2015)

Conocer la respuesta del organismo en el paciente quemado le permite al profesional de la salud comprender mejor la patología y ofrecer un mejor y adecuado manejo, reducir la posibilidad de complicaciones y obtener resultados más satisfactorios. Un tratamiento adecuado reduce significativamente la probabilidad de desarrollar IRA en el paciente quemado.

El objetivo del presente estudio consiste en compendiar de manera específica las diferentes alteraciones que pueden producirse en el sistema renal del paciente quemado, incluyendo la insuficiencia renal aguda (IRA), así como su manejo y monitoreo con el propósito de evitar mayores complicaciones.

\section{Materiales y métodos}

Con la finalidad de realizar el presente estudio se llevó a cabo una búsqueda para recolectar y seleccionar el material bibliográfico digital, que fue la base para analizar y compendiar el tema de los pacientes quemados y la insuficiencia renal aguda (IRA). Razón por la cual la presente investigación se clasifica como de tipo documental o bibliográfica.

Fueron usadas varias bases de datos y páginas web relacionadas con el área de la salud de carácter nacional e internacional, con validez científica y reconocida, para ubicar el material que sirvió como plataforma para el estudio. Estas bases de datos fueron las siguientes: SciELO, RECIMUNDO, Redalyc, Medigraphic, entre otros; y páginas como Manuales MSD, Organización Mundial de la Salud (OMS), entre las principales. El tipo de material bibliográfico para la selección consistió en protocolos, informes, tesis de grado, guías clínicas, y otras clases de contenidos. Los contenidos repetidos fueron desestimados, así como editoriales o cartas editoriales, anotaciones académicas y cualquier otro tipo de material bibliográfico carente de fuentes de sustento científico o con bajo nivel de evidencia. La búsqueda fue llevada a cabo durante el mes de septiembre del presente año, se utilizaron los siguientes descriptores: "paciente quemado", "insuficiencia renal aguda"; "quemaduras" e "insuficiencia renal aguda en el paciente quemado". Los registros bibliográficos ubicados fueron filtrados en base a los criterios de idioma español, relevancia del tema, con una correlación temática y actuales (fecha de publicación de los últimos 10 años). Cabe destacar que tanto la metodología como el análisis y la argumentación que aquí se expone se llevó a cabo de manera consensuada entre los miembros del equipo investigador.

\section{Resultados}

\section{Cómo se producen las alteraciones del sistema renal en los pacientes quemados}

Para Calderón Martínez (2013) el desarrollo y gravedad de las complicaciones del paciente quemado, son directamente proporcionales a la magnitud de la quemadura. Igualmente es importante destacar que hasta no estar definitivamente cerrada la herida existe riesgo de desarrollar complicaciones. Las iatrogenias son causas importantes de complicaciones, las cuales pueden presentarse por causa de reacciones adversas de medicamentos o tratamientos. Cuando se presentan complicaciones estas aumentan el estrés del paciente. "El fallo renal puede presentarse primero en pacientes con enfermedad renal intrínseca o en aquellos sometidos a periodos prolongados de choque hipovolémico, fallo hepático en pacientes con cirrosis o fallo cardíaco por enfermedad miocárdica de base". (p. 14)

Las quemaduras causan afectación tanto a nivel local como a nivel sistémico, uno de los principales órganos afectados en el paciente quemado y con un importante índice 
de morbimortalidad son los riñones. Cachafeiros Fuciño (2017) explica, con relación la forma y momento en que se pueden originar fallas en este sistema en el paciente quemado, lo siguiente:

El fracaso renal que se observa durante los primeros días, suele ser de causa prerrenal por hipoperfusión, por eso la diuresis es un buen indicador del flujo sanguíneo renal, y suele evitarse con una adecuada y precoz reanimación inicial. A partir de la segunda semana la etiología del fracaso renal se relacione más con patología propia del riñón, como la necrosis tubular aguda, en relación con nefrotóxicos o la sepsis. El desarrollo de fracaso renal se relación con un importante aumento de la mortalidad. (p. 40)

Alcivar Villavicencio (2015) con relación a las alteraciones renales en el paciente quemado refiere que "en los túbulos renales aparecen depósitos de hemoglobina con degeneración parenquimatosa difusa y signos de fallo renal que puede llegar a la anuria. En los casos más graves aparece necrosis cortical bilateral, nefritis intersticial, abscesos múltiples e infartos difusos". (p. 7)

En los riñones del paciente quemado se producen importantes alteraciones que compromete este sistema y su función, aumentando las probabilidades de morbimortalidad. Estas alteraciones se producen de la siguiente manera:

Los pacientes quemados presentan una disminución del flujo renal y de la tasa de filtración glomerular, debido a una disminución del volumen sanguíneo y del gasto cardiaco. La angiotensina, la aldosterona y la vasopresina también provocan disminución del flujo renal. Estos cambios Ilevan al paciente a oliguria, que de no ser tratada puede producir necrosis tubular aguda y falla renal. La hemólisis cuando es extensa, o la rabdomiolisis en las quemaduras eléctricas, dan lugar a depósitos de hemoglobina y mioglobina en el túbulo renal, ocasionan- do taponamiento de los mismos con posterior necrosis tubular aguda e insuficiencia renal aguda (IRA). El desarrollo de fórmulas efectivas para la resucitación del paciente quemado ha sido uno de los triunfos más importantes alcanzados en el tratamiento en los últimos años. Se debe mantener un gasto urinario entre 50 y $70 \mathrm{cc} /$ hora en los adultos y de $1 \mathrm{cc} / \mathrm{Kg} /$ hora en los niños, el monitoreo de la diuresis debe ser estricto especialmente durante las primeras 72 horas postquemadura. La resucitación temprana disminuye la incidencia de falla renal y de mortalidad. (Ramírez, Ramírez B., González, Ramírez, \& Vélez, 2010, p. 60, 61)

Para Casal (2017), la insuficiencia renal aguda (IRA) es el resultado de una reducción del gasto cardíaco y del aumento en la secreción de mediadores inflamatorios (angiotensina, aldosterona y vasopresina). En cuanto a las estadísticas este autor refiere: Está bien claro que la IRA se encuentra relacionada con un aumento de la mortalidad en pacientes con grandes quemaduras, tanto adultos como pediátricos (88\% y $55 \%$, respectivamente). En un estudio retrospectivo de Mustonen de 238 grandes quemados admitidos en una unidad de cuidados intensivos, la IRA ocurrió en 39\% y 1 de cada 3 de estos pacientes requirió un trasplante renal. El porcentaje de SCQ era significativamente mayor en pacientes que desarrollaron IRA comparado con aquellos que no la desarrollaron (40,2\% vs. $25,7 \%$ ). La mortalidad fue significativamente superior en pacientes que desarrollaron IRA comparados con aquellos que no $(44,1 \%$ [41 de 93 pacientes] vs. 6,9\% [10 de 145 pacientes]). Sumado a eso, para aquellos pacientes que se requirió trasplante renal $(n=32)$, la mortalidad fue superior en aquellos que desarrollaron IRA debido a mal manejo inicial comparados con aquellos que desarrollaron IRA de manera tardía $(66,7 \%$ vs. $57,1 \%$ ). (p. 62)

\section{Manejo del Paciente quemado con res- pecto a la IRA}

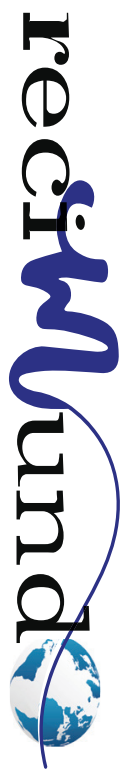


Para el manejo adecuado del paciente quemado es importante determinar si existen patologías previas. En el caso que nos ocupa, los pacientes con patologías renales tienen mayor riesgo de empeorar su condición, en virtud de lo cual es determinante para establecer el tratamiento adecuado, conocer esta situación. En el caso de este tipo de patología pre existente, se intentará, en primer lugar, la reanimación por vía oral siempre que se pueda. Es importante la reanimación con líquidos intravenosos en este tipo de pacientes. Entre las consideraciones especiales para la reanimación en pacientes con quemaduras eléctricas y con traumas por aplastamiento asociados, con padecimiento de mionecrosis y mioglobinuria que llevan a IRA, el tratamiento debe ser agresivo y requieren volúmenes mayores de la fórmula de resucitación a los calculados. "Se les debe mantener la diuresis entre 75$100 \mathrm{~mL} /$ hora utilizando, en caso de necesidad, diuréticos osmóticos (manitol 12,5-25 g). En los pacientes con falla renal el manejo de líquidos no puede regirse por la diuresis". (Rodríguez \& Miquet, 2010)

Rosanova et al. (2019), en cuanto al tratamiento de falla renal en el paciente quemado es importante considerar lo siguiente:

La insuficiencia renal aguda como complicación de la sepsis disminuye la eliminación de antimicrobianos como ß-lactámicos, vancomicina, aminoglucósidos y quinolonas, generando concentraciones plasmáticas más elevadas. En caso de antibacterianos como aminoglucósidos o vancomicina, puede dar origen a toxicidad ya que tienen un margen terapéutico estrecho, por lo que la dosificación de estos fármacos necesita ajustarse a la función renal. Debido a los cambios farmacocinéticos los quemados necesitan dosis diferentes de antimicrobianos, ya sea aumentando la dosis o disminuyendo los intervalos de administración. En pacientes con función renal disminuida se requiere disminuir la dosis para prevenir la acumulación de droga. Es recomendable ajustar los ATB en el paciente quemado con estudios farmacocinéticos, algunos de los cuales están disponibles (especialmente aminoglucósidos, vancomicina, ceftazidime, imipenem, aztreonam). (p. 20, 21)

Es importante considerar para la administración del tratamiento que la exposición a un sin número de agentes terapéuticos, puede interferir con la función renal y producir nefropatía. Un ejemplo de estos son drogas neurotóxicas tales como: amino glucósidos, penicilina, cefalosporinas, amfotericina, furosemida y tiázidicos, los AINES, nitrato de plata y terapia de reanimación con salino hipertónico. En la IRA relacionada con sepsis, existe interferencia con el volumen sanguíneo efectivo circulante y perfusión renal lo que origina azotenia prerenal. En los casos en que no se puede restablecer la circulación por terapia antimicrobiana adecuada y expansores plasmáticos, la necrosis tubular aguda secundaria, sobreviene rápidamente, a isquemia. El tratamiento de la insuficiencia renal en el período pos-quemadura incluye lo siguiente:

- Suplementos calóricos y proteicos que reduzcan el estado catabólico, disminuyendo de esta forma los productos nitrogenados (urea) y estabilización de zlectrolitos.

- Diálisis o ultrafiltrado lento, que puede ser hemofiltración arteriovenosa continua, hemodiálisis intermitente o diálisis peritoneal. La diálisis, en cualquiera de sus variantes es de mal pronóstico en un paciente quemado por demás con estado hipermetabólico e hiperdinámico máximo. (Casal, 2017, p. 62)

Por último, Nebot Fernández (2016), destaca otro aspecto importante a considerar para el tratamiento del paciente quemado y las alteraciones del sistema renal, relacionado específicamente con las alteraciones en el sistema urinario, las cuales pueden ser solventadas mediante "una adecuada reposición de líquidos y el uso de diuréticos. Finalmente, se restaura el equilibrio hí- 
drico mediante la disminución del edema, el retorno del líquido intersticial al espacio vascular y las diuresis en las que se pierde sodio y potasio". (p. 11)

\section{Las pruebas de función renal y el monito- reo en el paciente quemado}

Las pruebas de función renal, por lo general, están diseñadas para situaciones comunes. No obstante, para catástrofes metabólicas se deben considerar muchos otros factores. Ferrada, Tróchez, \& Ayala (2015), considera los siguientes factores:

- El nitrógeno ureico y la creatinina suben cuando 2/3 partes del parénquima renal está dañado (si se habla de daño renal).

- El sodio en la orina varía por cambios de flujo medular o tubular, por cambios hormonales, o por las soluciones intravenosas usadas.

- El volumen de orina cambia mucho, y está aumentado en insuficiencia renal de alto gasto.

- La gravedad específica urinaria no es confiable. Si se dan proteínas es isoosmolar con el plasma.

- La osmolaridad urinaria está alterada cuando hay pérdida de sustancias moleculares mayores en la orina y varía por la cantidad de agua ingerida antes de la determi $\neg$ nación. Al evaluar la función renal se debe tomar en cuenta la gravedad específica y la osmolaridad urinaria, si ambas están altas quiere decir que el riñón funciona, que tiene capacidad de concentración.

- Aclaramiento de agua libre: Sube más de 100 y luego baja a cero tres días antes de que se presente la insuficiencia renal.

$\mathrm{C} \mathrm{H} 2 \mathrm{O}=\mathrm{V}$ (volumen orina $)-\mathrm{C}$ osmolaridad (aclaramiento osmolar)

$\mathrm{C}$ osmol. $=$ osmol.urinaria $\times$ volumen orina osmol. plasmática

Lo normal es negativo $=-25 \mathrm{a}-100 \mathrm{cc} /$ hora . La relación osmol.urinaria / osmol. plasmá- tica de más de 1.7 indica buena capacidad de concentración. Esta fórmula puede predecir con 95\% a 98\% de certeza una insuficiencia renal. Para obtener la osmolaridad plasmática se pide el examen al laboratorio o se usa esta fórmula:

$2 \mathrm{Na}+$ glucosa + BUN = OSM. PLASMÁTICA. (p. 291)

\section{Conclusiones}

La IRA es una alteración del sistema renal que puede estar presente como patología pre existente en el paciente quemado o que puede producirse como consecuencia de las alteraciones sistémicas a las que es sometido el organismo tras la quemadura. Ambas condiciones son de vital importancia para el establecimiento de un manejo adecuado y reducir la tasa de morbimortalidad con respecto a estos pacientes.

Existen diferentes alteraciones en el sistema renal como consecuencia de las quemaduras, sin embargo, el desarrollo de fracaso renal, en cualquiera de sus grados, se relación con un importante aumento de la mortalidad.

Con relación al manejo de los pacientes quemados para evitar o tratar las alteraciones renales, específicamente la IRA, es importante en primera instancia la reanimación con líquidos ya sea por vía oral o endovenosa. Con relación a los antibióticos o cualquier otro fármaco, es necesaria la valoración y consideración de los daños causados al sistema renal, con la finalidad de establecer las dosis y los intervalos y evitar otras complicaciones.

\section{Bibliografía}

Alcivar Villavicencio, E. D. (2015). Síndrome de respuesta inflamatoria en niños quemados. Propuesta para su prevención y manejo. Tesis de Grado - Magister, Universidad de Guayaquil, Facultad de Ciencias Médicas, Guayaquil. Recuperado el 30 de Agosto de 2020, de http://repositorio.ug.edu. ec/bitstream/redug/9655/1/TESIS\%20FINAL.pdf 
Cachafeiros Fuciño, L. (2017). La influencia del síndrome de inhalación en la evolución y el pronóstico del paciente quemado crítico. Tesis Doctoral, Universidad Autónoma de Madrid, Facultad de Medicina. Departamento de Cirugía, Madrid. Recuperado el 10 de Septiembre de 2020, de https://repositorio.uam.es/bitstream/ handle/10486/680234/cachafeiro_fucinnos_lucia. pdf?sequence $=1$ \&isAllowed $=y$

Calderón Martínez, J. C. (2013). Quemaduras principales complicaciones y factores relacionados. Tesis de grado, Universidad Veracruzana, Servicios de Salud de Veracruz, Veracruz. Recuperado el 18 de Agosto de 2020, de https://www.uv.mx/blogs/ favem2014/files/2014/06/PROTOCOLO-Cesar.pdf

Casal, I. (2017). Complicaciones a corto y largo plazo de grandes quemaduras térmicas y su tratamiento inicial. Revisión de la literatura. Revista Argentina de Quemaduras, 27(2), 58-70. Recuperado el 10 de Septiembre de 2020, de http://www.fundacionbenaim.org.ar/raq/RAQ-08-2017. pdf\#page $=20$

Ferrada, R., Tróchez, J. P., \& Ayala, J. (2015). Manejo del paciente quemado. En F. Salamandra, \& L. Quintero (Ed.), Trauma. Abordaje inicial en los servicios de urgencia (Quinta ed., pág. 676). Cali, Colombia: Fundación Salamandra. Recuperado el 10 de Septiembre de 2020, de https://biblioteca. salamandra.edu.co/MATERIAL\%20ACADEMICO/ LIBRO\%20TRAUMA\%20ULTIMO\%205\%20EDICCION/TRAUMA\%20COMPLETO\%20-\%2OPEQ.pdf

García Velásquez, L., \& Donis Barrera, X. (2014). Mortalidad en pacientes con quemaduras. Tesis de Maestría en Pediatría, Universidad de San Carlos de Guatemala, Facultad de Ciencias Médicas. Recuperado el 30 de Agosto de 2020, de http:// biblioteca.usac.edu.gt/tesis/05/05_9249.pdf

Malkina, A. (Agosto de 2019). Manuales MSD. Recuperado el 15 de Agosto de 2020, de https:// www.msdmanuals.com/es-ve/professional/trastornos-urogenitales/lesi\%C3\%B3n-renal-aguda/ Iesi\%C3\%B3n-renal-aguda-Ira?query=Lesi\%C3\%B3n\%20renal\%20aguda

Nebot Fernández, C. (2016). Importancia del papel de la enfermería en el abordaje inicial y la prevención de las quemaduras en niños producidas en el hogar. Tesis de Grado, Universidad de Cantabria, Escuela Universitaria de Enfermería "Casa de Salud Valdecilla". Recuperado el 30 de Agosto de 2020, de https://repositorio.unican.es/xmlui/ bitstream/handle/10902/8254/Nebot\%20Fernandez\%20C.pdf?sequence=1\&isAllowed=y

Organización Mundial de la Salud - OMS. (06 de Marzo de 2018). Organización Mundial de la Salud - OMS. Recuperado el 06 de Septiembre de 2020, de https://www.who.int/news-room/fact-sheets/detail/burns

Ramírez, C. E., Ramírez B., C. E., González, L. F., Ramírez, N., \& Vélez, K. (2010). Fisiopatología dfel paciente quemado. Revista de la Universidad Industrial de Santander, 42(1), 55-65. Recuperado el 05 de Septiembre de 2020, de https://www.redalyc.org/pdf/3438/343835697007.pdf

Rodríguez, R., \& Miquet, L. M. (2010). Atención Inicial al Paciente Quemado. Cirugía Reconstructiva y Quemados. INFOMED. Recuperado el 29 de Agosto de 2020, de https://files.sld.cu/cirugiareconstructiva/files/2010/08/atencion20inicial20al20paciente20quemado4.pdf

Rosanova, M. T., Mussini, M. S., Isasmendi, A., Pinheiro, J. L., Hernández, C., Laborde, S., \& Álvarez, V. T. (2019). Manejo de la Infección en Niños Quemados. Guía de atención pediátrica, Hospital de Pediatría Garragan, Coordinación de Evaluación de Tecnología Sanitaria. Recuperado el 01 de Septiembre de 2020, de https://www.garrahan.gov.ar/ images/intranet/guias_atencion/GAP_2010_-_MANEJO_INFECCION_QUEMADURAS_-_ACT.2019. pdf

Sanchez, E. E., Barrazueta, M. J., Cisneros, M. N., \& Vaca, D. A. (2019). Insuficiencia renal aguda en pacientes quemados. Recimundo, 3(3), 159-179. Recuperado el 08 de Septiembre de 2020, de http://recimundo.com/index.php/es/article/download/595/803/

Valdés, S., Palacios, I., \& Mariño, J. A. (2015). Tratamiento integral del paciente gran quemado. Revista Cubana de Medicina Militar, 44(1), 130-138. Recuperado el 12 de Septiembre de 2020, de https://www.medigraphic.com/pdfs/revcubmedmil/ cmm-2015/cmm151p.pdf

Wolf, S. E. (Noviembre de 2018). Manuales MSD. Recuperado el 30 de Agosto de 2020, de https://www. msdmanuals.com/es/professional/lesiones-y-envenenamientos/quemaduras/quemaduras 


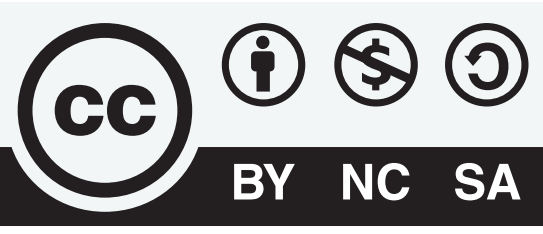

CREATIVE COMMONS RECONOCIMIENTO-NOCOMERCIAL-COMPARTIRIGUAL 4.0.

\section{CitAR ESTE ARTICULO:}

Zambrano Andrade, G., Lascano Torres, M., Galán Velasco, A., \& Sinche Cruz,

D. (2020). El paciente quemado y la insuficiencia renal aguda (IRA). RECI-

MUNDO, 4(4), 13-21. doi:10.26820/recimundo/4.(4).octubre.2020.13-21 